CORRESPONDENCE

\title{
Correspondence on statistical rigor and kappa considerations: which, when, and clinical context matters
}

Pediatric Research (2020) 88:6; https://doi.org/10.1038/s41390-0200880-z

The purpose of the study by Pavageau et al., published in Pediatric Research, 2020, ${ }^{1}$ was to determine the reliability of the modified Sarnat neurologic examination in late and moderately preterm neonates born at 32-36 weeks of gestational age. The authors concluded that the reliability of the neurologic exam between the gold standard (GS) study investigator and groups of attending neonatologists was good to excellent $(k>0.72)$ in most categories, except for Moro and tone in 34-36-week prematures, while the agreement was poor/fair for both tone and Moro categories in infants born at 32-34 weeks' gestation $(k=0.20-0.60)$. The authors report an unweighted Kappa statistic on which they based their conclusions. In a Letter to the Editor, ${ }^{2}$ it was pointed out that such a conclusion may have been due to the inappropriate use of the Kappa Statistic in place of the weighted Kappa, and this was supported by detailing certain statistical conditions to consider when choosing which results to report.

Choosing the correct statistical test to use and report can be a daunting task for both the statistician and the clinician. The statistician will tend to focus on mathematical assumptions of the test and how well the data meet those assumptions. The clinician on the other hand may focus on how the results of the test employed could be used in practice. The statistician will consider how the test quantifies the probability of error, either Type I or Type II, while the clinician may consider the consequence to the patient of an incorrect action. Is having a treatment without the disease in question worse than not having a treatment when the disease in question is present. The clinician would choose the test that minimizes the chance of the more consequential error, assuming of course that no statistical assumptions are violated. In this case, the choice is between a weighted or an unweighted Kappa Statistic. Other similar situations can be noted. Consider which is the more appropriate statistic: a positive predictive value (when a false positive is detrimental), versus a negative predictive value, when a false negative is detrimental. When planning and powering a study, recognizing the effect of Type I or a Type II error to the subjects is done by choice of the Alpha level and the Power.
Should the Alpha level be small $(<0.05)$, while the power remains standard (e.g., 80\%), or the power be high $(>=90 \%)$ while the Alpha level remains at 0.05 .

We have here a good example of these differing approaches. The authors state:

"While we agree with the general value of weighted kappa statistics and the importance of monitoring partial agreement, we have purposefully selected to use the conservative non weighted Kappa statistic in this particular clinical situation. The decision is based clinically on the need to completely agree on what constitutes moderate/severe encephalopathy. In such situations, a near miss is not acceptable, you either categorically initiate hypothermia or you don't. This determined the use of a statistical approach of complete agreement amongst gold standard and other examiners."

This is a supportable decision on their part. The comments provided in the letter support the more strictly statistical approach and are not wrong, but not as well-suited to these data as the approach the authors chose.

\section{ADDITIONAL INFORMATION}

Competing interests: The authors declare no competing interests.

Publisher's note Springer Nature remains neutral with regard to jurisdictional claims in published maps and institutional affiliations.

Mary Ann O'Riordan ${ }^{1}$ and Cynthia Bearer ${ }^{1}$

${ }^{1}$ Rainbow Babies \& Children's Hospital, University Hospitals of Cleveland, Cleveland, $\mathrm{OH}, \mathrm{USA}$ Correspondence: Mary Ann O'Riordan (Maryann.O'Riordan@UHhospitals.org)

\section{REFERENCES}

1. Pavageau, L. et al. Inter-rater reliability of the modified Sarnat examination in preterm infants at 32-36 weeks' gestation. Pediatr. Res. 87, 697-702 (2020).

2. Maleki, S. \& Naderi, M. Methodological issues on interrater reliability of the modified Sarnat examination in preterm infants. Pediatr. Res. 87, 614 (2020). 